\title{
LA RALENTIZACIÓN DE LA ECONOMÍA MUNDIAL
}

\author{
Beatriz Herrera Garcáa \\ Docente-Faculiad de Ciencias Contables
}

\begin{abstract}
RESUMEN
El fuerte crecimiento de la economía mundial entre los años 2003 y 2004 se reflejó en el acelerado crecimiento del comercio internacional, que a su vez impulsó al crecimiento económico. Algunos de los beneficiarios de este gran auge fueron los países en desarrollo exportadores de commodities (especialmente aquellos exportadores de energía y minerales). Es el caso del fuerte impacto provocado en algunas economías de la región nuestra, como Chile, Perú y Venezuela (exportadores de minerales los primeros y de energía el último), en los que el crecimiento registró tasas elevadas en comparación con la media latinoamericana (Chile, 5.8\% y Perú, 5.1\% del PBI en 2004).

Estados Unidos, China y los países en desarrollo de Asia Oriental y Nororiental y Asia Sudoriental, siguieron siendo los principales propulsores del crecımiento de la economía mundial (veáse Cuadro 1). En general, el crecimiento tuvo base amplia, apoyado por un fuerte impulso de las exportaciones y los precios elevados de los productos básicos, así como por una fuerte demanda interna, fomentada por la baja tasa de interés. Al igual que en el año 2003, la zona del euro siguió rezagada respecto de la recuperación mundial.
\end{abstract}

En este período se evidenció un hecho trascendental: China estaba transformándose en el tercer país en más importante en el comercio mundial. El rápido crecimiento de China ha sido fundamental en este proceso (el crecimiento más autónomo de los países de la CESPAP'), y las perspectivas para el año 2005 estarán condicionadas por el éxito de las autoridades chinas en lograr un «aterrizaje suave».

Palabras clave: Crecimiento económico, ralentización económica, comercio internacional, política económica.

Comisión Económica y Social para AsiayelPacífioo. Lospaíses dela CESPAPcrecieronen el 2004 a ritmo más rápido desde el 2000, con uncrecimientode base amplia acompañado, en la mayoría deloscasos, por bajainflación, en parte comoconsecuenciadelossubsidios al combustible. El crecimientofueimpulsadoporelaumentodelasexportaciones, gran parte de las cualesfueron intrarregionales, en particular hacia China. 
Cuadro N. ${ }^{\circ} 1$

El crecimiento económico en las principales regiones del mundo

(Variación porcentual)

\begin{tabular}{|l|c|c|c|}
\hline Países y regiones & 2003 & 2004 & 2005 \\
\hline America del Norte & 3,0 & 4,3 & 3,5 \\
\hline Estados Unidos de América & 3,0 & 4,4 & 3,5 \\
\hline Canada & 2,0 & 2,7 & 3,0 \\
\hline America Latina y el Caribe & 1,8 & 5,8 & 4,5 \\
\hline Europa Occidental, Central y Oriental & 1,1 & 2,4 & 2,2 \\
\hline Japon & 1,3 & 2,5 & 1,5 \\
\hline China & 9,3 & 9,5 & 8,5 \\
\hline India & 8,5 & 6,9 & 7,2 \\
\hline Rusia & 7,3 & 7,1 & 6,3 \\
\hline Passes de la CESPAP & 6,3 & 7,2 & 6,2 \\
\hline Asia Oriental y Noroccidental & 6,2 & 7,5 & 6,3 \\
\hline Asia Septentrional y Central & 7,6 & 7,2 & 6,5 \\
\hline Países Insulares del Pacífico & 3,0 & 3,0 & 2,6 \\
\hline Asia Meridional y Suboccidental & 7,2 & 7,4 & 6,4 \\
\hline Asia Sudoniental & 4,9 & 6,4 & 5,7 \\
\hline
\end{tabular}

Fuente: WorldEconomicSituation andProspects, 2005. Eurostat, CuentasNacionales y Estadísticas Nacional dela OCDE, 2004. Los datos para el 2005 corresponden a pronósticos.

\section{INTRODUCCIÓN}

Las perspectivas para el año 2005 indican una desaceleración (ralentización) del crecimiento económico en todas las regiones en desarrollo. A medida que se debilita el entorno externo, Europa Occidentaly Américadel Norte disminuirán su crecimiento de $3,3 \%(2005)$ a $2,8 \%$ (2006), con un aumento de la inflación, a medida que los precios más elevados del petróleo afectan a la economía en general.

La ralentización del crecimiento económico mundial en el año 2005 situará a la economía por debajo de la tasa del 2004 (véase Cuadro N. 1). Sin embargo, el proncipal impulso del crecimiento del comercio mundial seguirá dependiendo de China y EE.UU.

En la economía norteamericana se prevé una disminución del PBI real de 4,4\% en el año 2004 a 3,5\% en el año 2005. En general, la actividad económica de Estados Unidos se apoyará en la demanda interna, que será más moderada en relación al año anterior. Las exportaciones mejorarán debido a la depreciación del dólar y a la fuerte demanda externa registrada en los mercados de EE.UU., Asiay AméricaLatina; sin embargo,

Assa oriental y Nororiental: China, Hong Kong, Mongolia, Corea del Sur y Taiwán. Asia Septentrional y Central: Armenia, Azerbaryán, Georgia, Kazajstán, Kırguistán, Rusıa, Tayıkistán, 'Turkmenıstány Uzbekistán Paises Insulares del Pacifico: Islas Cook, Fiji, Papua Nueva Guinea, Samoa, Islas Salomón, Tongay Vanuatu AsıaMerıdıonal y Suboccidental: Bangladesh, Bhután, India, Irán, Nepal, Pakistán; Sri Lankay Turquía. Asia Sudorıental: Camboya, Indonesia, RDP de Lao,Malasia, My anmar, Filipinas, Singapur, Tailandia y Vietnam 
las importaciones crecerán también, y por ello el cambio en las exportaciones netas reales (exportaciones menosimportaciones) seguirá siendo un freno para la actividad económica estadounidense.

Un tema de grave preocupación es el aumentodelosdesequilibrios externosentre las principales economías y regiones del mundo. El gran déficit en la cuenta cor riente de la balanza de pagos de Estados Unidos siguió aumentando hasta alcanzar el 5,75\% del Producto Bruto Interno en el 2004. La principal consecuencia de ese déficit es el gran superávit registrado en las cuentas corrientes del Japón y las economías de reciente industrialización de Asia (NICs) y, en menor medida, de los países de la zona del euro (principalmente Alemania).

En el año 2004, el consumo privado estadounidense, favorecido por una lenta recuperación del mercado del trabajo, los bajos tipos de interés y el efecto riqueza creado por el auge del mercado inmobiliario, fue el principal elemento promotor de la actividad económica. La tasa de ahorro privado se mantuvo en un nivel muy bajo. El gasto de las empresas en equipo y material infor mático continuó pujante, estimulado por el significativo aumento de los beneficios de las empresas, una mayor tasa de utilización de la capacidad e incentivos fiscales. La inflación de los precios al consumidor aumentó un poco, en buena parte, debido a la subida del precio de la energía. El déficit en las cuentas comercial y corriente alcanzó un máximo histórico.

Dado el fuerte crecimiento económico, la reserva federal empezó a subir gradualmente el tipo de interés delos fondos federales (bonos del tesoro), aunque la política monetaria siguió apoyando claramente al crecimiento económico. Por otro lado, ese moderado ajuste a la política monetaria se vio compensado por una depreciación adicional del dólar, lo que creó condiciones monetarias muy favorables en el año 2004. Al mismo tiempo, los tipos de interés a largo plazo se mantuvieron muy bajos. Tras dos años de estímulos fiscales fuertes, la política fiscal fue solo ligeramente expansionista. El déficit presupuestario general del gobierno equivalió a más del $4,0 \%$ del Producto BrutoInterno nor teamericano en el año 2004.

Por otro lado, el año pasado, en la zona del euro, ${ }^{3}$ la recuperación económica perdió impulso. Elcrecimiento delas exportaciones, que había sido el principal propulsor de la recuperación, se debilitó debido a una cierta desaceleración de la economía mundial (a partir del segundo semestre de 2004) y a los efectos negativos de la apreciación del euro para la competitividad de los precios.

En la zona del euro, las inversiones de capital fijo aumentaron ligeramente, pero no adquirieron un impulso suficiente. Las inversiones fijas de las empresas se vieron frenadas por la incertidumbre en torno a las perspectivas de la demanda a mediano plazo y al rápido aumento de los precios del petróleo. En esta zona el PBI creció, en el 2004 , un 2,0\% respecto al año anterior. Ese promedio oscila entre un aumento del PBI real de poco más del 1,0\% en Italia, Holanda yPortugal, a un crecimiento del $4,0 \%$ y más en Irlanda y Luxemburgo. Alemania, la mayor economía de la zona del euro, alcanzó una tasa media positiva de $1,7 \%$, tras dos años de estancamiento.

3 Alemania, Austria, Bélgica, España, Finlandia, Francia, Grecia, Irlanda, Italia, Luxemburgo, PaísesBajos(Holanda) yPortugal(UE-12).Si sesumana estos: Dinamarca, Inglaterray Suecia, setienealaUnión Europeadelos 15 (UE-15). 


\section{LA IMPORTANCIA ECONÓMICO- POLITICA DE CHINA}

Con un crecimiento anual superior al $9,0 \%$ durante más de dos décadas, China tiene un peso económico en la economía mundial que no hadejado de aumentar. ${ }^{4} \mathrm{Con}$ un sector externo que se expande mucho más rigurosamente que el resto del mundo, Chınaestáintegrándose ala economíaglobal con rapidezymucha estrategia. Sin embargo, de acuerdo a la experiencia histórica, la rápida integración de China a la economía mundial puede redundar en una intensificación de la competencia internacional en varios sectores $y$ provocar cambios significativos en la asignación de recursos entre sectores y países. Al mismo ttempo, varias características relacionadas con el tamaño, la estructura y las instituciones chinas pueden contribuir a que las repercusiones globales de su crecimiento sean mayores a las que se produjeron en los casos históricos del Japón y de los NICs.

Históricamente, China fue una importante potencia económica durante los 18 primeros siglos de nuestra era y representaba aproximadamente el $20 \%$ de la economía mundial. En la actualidad, la participación de China en el Producto Mundial Bruto supera el 3,5\%; este resurgimientotal vez represente el comienzo de la vuelta de China a la posición que disfrutó durante largo tiempo.

El tamaño importa y ya se ha puesto de manifiesto. E.g., la demanda de materias primas y energía de China ha crecido exponencialmente en los últimos tiempos, impulsado por el extraordinario aumento de la demanda de vivienda y automóviles, ${ }^{5}$ por partedelosconsumidores y porlaexpansión de la inversión. Este factor, junto con algunos otros, ha hecho que los precios internacionales de muchos productos básicos alcancen niveles máximos sin precedentes en varios años.

Entrelos principales rasgos estructurales de la economía china, que explican su ventaja comparativa en la producción de bienes con gran densidad de mano de obra en la economía mundial, sobresalen una enorme masa de mano de obra escasamente cualificada, bajos costos laborales (en la industria manufacturera representa menos de un tercio de la de México) y escasez de tierra cultivable per cápita y altas tasas de ahor ro doméstico.

Por otro lado, mientras China recibe un gran porcentajede lascorrientes de inversión extranjera directa, su elevada tasa de ahorro interno indica que su dependencia de la financiación externa sería inferior a la de la gran mayoría de los países en desarrollo.

China, otrora economía de planificación centralizada, ha logrado avances en las últımas décadas en su transfor mación hacia una economía de mercado; sin embargo, el marco institucional de la economía china sigue siendo diferente de otras economías en lo que respecta al elevado porcentaje de la propiedad estatal, la participación del Gobierno en la adopción de decisiones económicas y sus sistemas jurídicos, bancario y financiero insuficientemente desarrollados.

\footnotetext{
ul en esa partedel mundo, con todas las consecuencias que ello acarrearía enel planoeconómico, comercial y político
} 
No obstante, la sostenibilidad del crecimiento de China, su ascenso en la economía global supone importantes desafíos para otras muchas economías. Ahora, tiene una posición cada vez más influyente en la economía mundial

\section{EL RESTO DE ECONOMÍAS EN DESARROLLO DE ASIA}

Es de destacar que las economías en desarrollo de la región asiática, agrupadas en la CESPAP, han pasado a ser exportadoras netas de capital a medida que los superávit persistentes de la cuenta corriente de la balanza de pagos de algunos países se han combinado con un aumento abrupto de las corrientes netas de capital privado, reflejadas principalmente en la acumulación sustancial de reservas oficiales.

Para esa región, en el 2005, se estima una devaluación del crecimiento del Producto Bruto Interno a un 6,2\% (veáse Cuadro $N^{\circ} 1$ ), a medida que se debilita el entorno externo, y se estima que la inflación se reducirá ligeramente, en el supuesto de queel precio mediodel petróleose mantenga alrededor de 38 dólares por barril.

En las diferentes subregiones de la CESPAP, el ímpetu del crecimiento ha respondido a cada economía. Así, el crecimiento general de Asia Oriental y Nororiental aumentóal 7,5\% en el año 2004, es decir, 1,3 puntos porcentuales superior al año 2003. Lainflación aumentóy seespera que siga elevada en el 2005. Las perspectivas de crecimiento de la subregión están íntimamente vinculadas con los efectos de las medidas que el gobierno de China está adoptando actualmente para desacelerar el crecimiento de su economía en el año 2005. Hay consenso en que la economía china debe desacelerarse gradualmente hasta alcanzar un ritmo sostenible y, posteriormente, habría de ampliar el espacio para que los países y economías de la subregión ajustaran sus políticas.

La subregión de Asia Septentrional y el Asia Central, en 2004, disfrutaron de otro año de alto crecimiento. Las perspectivas de la subregión para 2005 son de crecimiento moderado del producto y de la inflación a medida que los precios del petróleo y de otros productos básicos se reduzcan. El sector de la energía ha sido el principal impulsor del crecimiento en esta subregión, correspondiéndole aproximadamente un tercio del PBI y más de la mitad de las exportaciones en Rusia y Kazajstán. Sin embargo, el retraso de la inversión en oleoductos y capacidad portuaria y en general en la infraestructura energética ha impuesto un límite al crecimiento del sector.

En la subregión de Asia Meridional y Sudoriental el crecimiento mejoró marginalmente en el año 2004, a pesar del mal clima de Asia Meridional y el mayor costo del petróleo. El crecimiento del PBI del Asia Sudoriental superó en el mismo año las expectativas. El crecimiento tuvo así amplia base en la mayoría de los países y la manufactura, en particular, se benefició con la recuperación del ciclo electrónico y la fuerte demanda de exportación. El mayor precio de los alimentos y la energía hizo presión sobre el nivel de precios de la subregión, en parte compensado por los subsidios del combustible en algunos países. Si bien los desastres provocados por el terremoto y el tsunami redujeron las expectativas de crecimiento de Tailandia e Indonesia. Para la subregión en su conjunto se espera que la tasa de crecimiento del PBI se reduzca en 2005. Si bien el precio elevado 
de los productos básicos impulsó varias economías de la subregión en 2004 habiendose beneficiado Indonesia, Malasia y Vietnam con los precios más altos, el uso delossubsidios del petróleo en varios países, en particular, Indonesia, Malasia y Tailandia probablemente sean fuente de graves problemas fiscales.

\section{LOS PROBLEMAS A ENFRENTAR}

Se espera para el año 2005 una reducción del crecimiento económico mundial, así como del crecimiento del comercio internacional.Lademandade importaciones de los países en desarrollo probablemente no compensará la desaceleración de las economías desarrolladas, pero China seguirá determinando en medida importante el rendimiento comercial de la mayoría de los sectores de la región (CESPAP).

La economía mundial se sigue caracterizando por los grandes desequilibrios de la cuenta corriente y el menor valor del dólar. Este último contribuyó a reducir el déficit de EE.UU, pero muchas economías de la región de la CESPAP han dependido de la demanda estadounidense de importaciones como

cumunu consigerable

deı valor del dólar. Además, la reducción del valor del dólar impulsaría, a su vez, la inflacion en EE.UU., lo que provocaría mayores niveles de la tasa de interés estadounidense y mundial.

Coyunturalmente, la situación energética reviste connotaciones políticas significativas para los gobiernos de la región la misma que han agravado los precios del petróleo. Dicha situación, que ya era de volatilidad significativa, probablemente mantenga la presión al alza durante algún tiempo. Por otro lado, existe un claro riesgo de deterioro del entorno externo. Finalmente, todas esas transformaciones, unidas al debilitamiento del dolar, constituirán un problema importante para mantener el ritmo de crecumiento y la estabilidad macroeconómica.

\section{LA DINÁMICA DE LA ECONOMÍA MUNDIAL EN 2004}

Alentado por los bajos tipos de interés y las medidas de estímulo en los Estados Unıdos principalmente, así como por el rapido ascenso de China como importador importante y como fuente considerable de exportación, el crecimiento de la economía mundral aumentó en un $4,0 \%$ en el 2004 (véase Cuadro N. ${ }^{\circ}$ 2). Las tasas de crecimiento de los países en desarrollo, en general, aumentaron $5,5 \%$, tras la expansión que se produjo en las principales economías (vease Cuadro N. ${ }^{\circ} 1$ ). Sin embargo, la creacion de puestos de trabajo sigue siendo un problema en la mayoría de los países. Casi todas las economías experimentaron un aumento en el desempleo y en el subempleo en los ultımos años de desaceleración global, y el empleo va siempre a la zaga de otros aspectos de una recuperación.

No obstante, los constantes y cada vez mayores desequilibrios internacionales, que se expresan en el gran déficit externo de los Estados Unidos y los excedentes equiparables en unas cuantas economías también plantean una serie amenaza. La torma en que evolucionen esos desequilibrios tendrá consecuencias profundas para la estabilidad, la eficiencia y la equidad de la economía mundial en el tuturo. En este contexto, se debe evitar la tentación de aplicar medidas proteccionistas para reducir el deficit en cuenta corriente y proteger los 
puestos de trabajo. La creación de puestos de trabajo se ve entorpecida en gran medida por factores internos cíclicos, así como por la necesidad de emprender reformas estructurales en algunos países, más que por la transferencia de puestos de trabajo a países en desar rollo.

Un proceso de ajuste preferible extrañaría la adopción de políticas coordinadas para reducir las diferencias estructurales en materia de crecimiento entre las principales economías, así como la cooperación internacional para facilitar el crecimiento sostenido en los países en desarrollo, con lo que aumentaría en términos generales. En particular, es necesario que Estados Unidos reduzca su déficit gubernamental y que Europa y Japón adopten medidas para fomentar el crecimiento a largo plazo.

Sin embargo, los desequilibrios mundiales, y en particular el déficit de Estados Unidos, no se corregirá con la veloz caída del dólar norteamericano como se esperaba. Lacaída del dólar no estálogrando corregir el problema, pues Estados Unidos se encuentra en una posición única, en la que pueden mantener su deuda en su propia moneda, al ser el principal tipo de cambio para el comercio mundial. Parte de los efectos debilitadores asociados generalmente con la depreciación están siendo trasladados a los acreedores de Estados Unidos, los cuales sufren una pérdida de su patrimonio debido a que sus grandes tenencias de dólares van menguando su valor. Por lo que persiste la posibilidad de una cor rección abrupta y dañina a nivel mundial debido a los desequilibrios.

Por lo tanto, una respuesta mundial a los desequilibrios debería incorporar estímulos a la demanda nacional, preferentemente por medio de inversiones nacionalesen los países con grandes excedentes externos tales como Japón, Europa Occidental y algunos países en desarrollo de Asia. La reconstrucción necesaria luego del tsunami en diciembre pasado ofrece una oportunidad para realizar inversiones de gran escala en los países del Océano Indico.

En consecuencia, la recuperación económica cíclica ha alcanzado su máximo en el 2004, con un crecimiento del 4,0\%, y se prevé una disminución para 2005 , debido a la evolución del dólar y de los desequilibrios mundiales; la tasa estimada es de $3,25 \%$, debido a una falta de respuesta del mercado a las implicaciones de la caída del dólar y al aumento del déficit comercial.

\section{Cuadro N. 2}

Crecimiento de la producción y el comercio mundial (Variación porcentual)

\begin{tabular}{llllll}
\hline & 2000 & 2001 & 2002 & 2003 & 2004 \\
\hline Producción Mundial & 3,9 & 1,3 & 1,7 & 2,8 & 4,0 \\
Comercio Mundial & 11,5 & $-0,9$ & 2,5 & 6,2 & 10,6 \\
\hline
\end{tabular}

Fuente:Departamentode Asuntos Económicosy Sociales, 2004, Naciones Unidas. 
Sin embargo, las economías de China y los Estados Unidos, en conjunto, están sosteniendo el crecimiento mundial; el consumo de los Estados Unidos estimula al sector manufacturero mundial, mientras que la creciente demanda china de materias primas está mejorando el mercado de la exportación de productos básicos de los países en desar rollo.

La demanda china y el resurgimiento de la economía mundial han revertido la persistente declinación en los precios de los productos básicos. $^{6}$

El crecimiento de 5,5\% promedio, en el 2004 , en los países en desarrollo fue el más alto de las últimas dos décadas, impulsado parcialmente por el fortalecimiento de los mercados de productos básicos. La producción en los países de sudeste asiático también fue la más sólida desde que se inició su recuperación, luego de la crisis financiera de 1997.

En todas las regiones subdesarrolladas no se consiguió un crecimiento superior al $7,0 \%$, que se consideraba necesario para alcanzar los objetivos del "Desarrollo del Milenio".

El precio del petróleo aumentó un 5,0\% en la primera mitad del 2004, antes de caer nuevamente. Se espera que continúe la moderación de los precios durante el 2005. La razón es que el aumento del año pasado se debió a un crecimiento de la demanda y no a un déficit en la producción, como sucedió durante las perturbaciones en el mercado petrolero en el pasado, y por lo tanto, es más susceptible a ajustes suaves del mercado.

\section{LA ECONOMIA LATINOAMERICANA EN 2004}

La economía latinoamericana creció un 5,8\% en el año 2004. Para el año 2005 se prevé un crecimiento cercano al $4,5 \%$, debido a que el contexto internacional será menos favorable que el año anterior. Se estıma que el crecimiento mundial se situará en torno al 3,25\%, debido a los desequilibrios mundiales y a los efectos recesivos e inflacionarios del alto precio de petróleo.

Un hecho significativo del proceso de recuperación en comparación con su historia económica, es que por segundo año consecutivo el crecimiento del PBI se dio paralelamente a un saldo positivo de la cuenta corriente de la balanza de pagos. Otra característica distintiva es que el notable crecimiento del PBI se dio en un contexto de salida de capitales. Sin embargo, en los dos últimos años la fortaleza del sector externo per mitióacumular un saldo acreedor en las transacciones realizadas entre la región y el resto del mundo. El aumento de la tasa de crecimiento del PBI respondió en gran parte al dinamismodelas exportaciones. Por último, en general, se produjo un aumento de las reservas internacionales de la región. Es decir, el significativo repunte latınoamericano se explica a partir del 2003 $y$, sobre todo, en el 2004, por las favorables condiciones externas que comenzaron a influur positivamente en los tér minos de intercambio, lo que condujo a un aumento del poder de compra de las exportaciones y estimuló una expansión de su oferta, dada su mayor rentabilidad. De esta forma, las exportaciones se convirtieron en el motor 
inicial del proceso de recuperación del nivel de actividad, acompañados, sobre todo inicialmente, por un aumento de la oferta de productos nacionales sustitutivos de importaciones. Sin embargo, en el contexto de una elevada tasa de desempleo, bajos salarios reales, escasa utilización de la capacidad instalada y, en muchos casos, de antecedentes cercanos de acentuada inestabilidad, la demanda interna haya demorado en reaccionar.

Las perspectivas para el 2005 son una ralentización de las economías que el año anterior propulsaron el crecimiento mundial, por lo que los estimados para la producción y el comercio mundiales serán menores, afectando de distinta manera a los países en desarrollo beneficiarios de esta expansión.

\section{REFERENCIAS}

Banco Mundial. Flujos Mundiales de Financiamiento para el Desarrollo. Infor me Anual, Washington, 2004.

Consejo Económico y Socialo Resumen del Estudio económico de Europa de 2005: la situacióneconómucade Europayla Comunidad. de Estados Independientes en 2004-2005. Naciones Unidas, Nueva York, 2004. - - . Resumen del Estudio Económico de Amérza Latina yel Caribe 2004. Naciones Unidas, Nueva York, 2004.

- - Economic and Social Survey of Asia and the Pacific 2005. Naciones Unidas, Nueva York, 2004.

DESA-UNCTAD. WorldEconomic Situation and Prospects 2005. Naciones Unidas, Nueva York, 2004.

Economic and Social Council. World Economic and Social Survey 2004. Naciones Unidas, Nueva York, 2004., Krugman, P.R. y M. Obstfeld. Economía Internacional, Teoría y política. Pearson Educación S. A., 2001.

Tugores, J. Economía Internacional, Globalzzación e Integración Regional. Mc Graw Hill, 2002.

Yang Yongzheng. Cbinas Integration into the World Economy: Implications for Derdoping Coumtries. FMI, Washington, 2003.

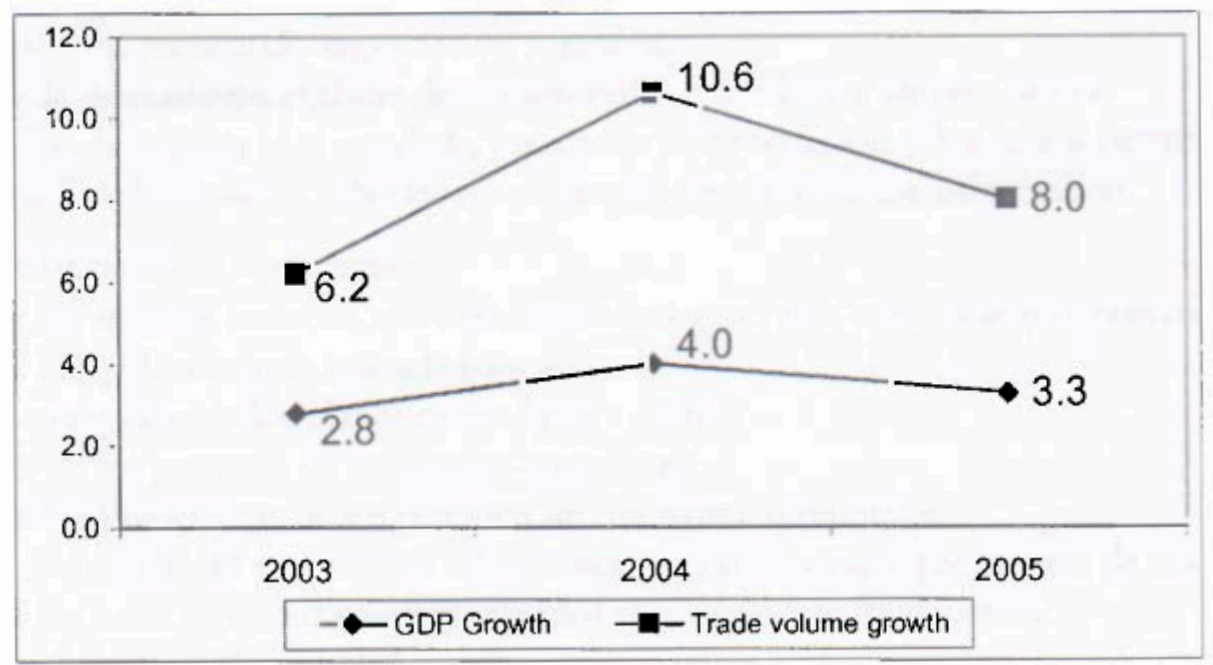

Gráfico N. ${ }^{\circ}$. El crecimiento global y el comercio internacional en 2005. 